\title{
Transperitoneal laparoscopic treatment for recurrence of a giant multilocular prostatic cystadenoma: A case report and review of the literature
}

\author{
Davide Abed El Rahman ${ }^{1}$, Tiziano Zago ${ }^{1}$, Giuseppe Verduci ${ }^{1}$, Gianpaolo Baroni ${ }^{1}$, \\ Marco Lorenzo Berardinelli ${ }^{1}$, Umberto Pea ${ }^{2}$, Eugenio Morandi ${ }^{3}$, Marco Castoldi ${ }^{3}$ \\ ${ }^{1}$ Urology Department, "Guido Salvini” Hospital, ASST Rhodense, Rho (MI), Italy; \\ ${ }^{2}$ Urology Department, "Fatebenefratelli e Oftalmico" Hospital, ASST Fatebenefratelli Sacco, Milano, Italy; \\ ${ }^{3}$ Surgery Department, "Guido Salvini" Hospital, ASST Rhodense, Rho (MI), Italy.
}

\begin{abstract}
Summary Giant multilocular prostatic cystadenomas (GMPC) are very rare benign tumors that originate from the prostate with extensive spread into the pelvis. The lesion may present as large abdominal mass causing obstructive voiding dysfunction and usually not invading adjacent structures. All of the previously reported patients with GMPC underwent open surgery. Although the natural history of prostatic cystadenoma remains unknown, complete surgical excision may not always be necessary. We report the case of a 74-year-old male who presented a retrovesical recurrence of prostatic cystoadenoma after 16 years, treated with a laparoscopic approach. To our knowledge this is the first case of laparoscopic management of GMPC. In this article we review the current literature about this rare tumor and discuss the diagnostic and management dilemmas posed by this rare pathologic condition. We believe that physicians should at least be aware of the existence of this disease in the differential diagnosis of pelvic cavity tumours and, considering the benignity of GMPC, they should propose -as first- $a$ minimally invasive approach.
\end{abstract}

KEY WORDS: Cystadenoma; Prostate; Multilocular; Laparoscopy. Submitted 11 July 2015; Accepted 11 September 2015

\section{INTRODUCTION}

Giant multilocular prostatic cistoadenoma is a rare benign tumor of the prostate gland $(1,2)$; it is usually characterized by large multilocular cysts located between rectum and bladder, attached to the prostate via a pedicle or, rarely, entirely separated from the prostate. It is not always possible to determine the exact point of origin of these multilocular cystic neoplasms because of their large size at the time they are diagnosed.

The clinical presentation includes obstructive voiding symptoms such as poor stream, intermittency, sensation of incomplete emptying, acute urinary retention and sometimes constipation symptoms due to mechanical compression of the lower intestine $(1,3)$. The age of patients ranges from 15 (6) to 80 (1) years.

Microscopically it is characterized by typical prostatic glands and cysts lined with double layers of columnar and cuboid cells with basally located nuclei and pale cytoplasm.

The epithelial cells usually show positive prostatic specific antigen (PSA) staining (4).

Different therapeutic approaches can be employed since some authors describe total surgical excision as therapy of choice (5) due to the risk of recurrence in case of partial resection, while many others think that radical surgery is not necessary (6); it is also described a "medical" treatment using GnRH antagonists with excellent results (3). We report the first case - to our knowledge - of laparoscopic treatment for a recurrent giant multilocular prostatic cystadenoma.

\section{Case report}

We report the case of a 74 years old patient - with an history of hypertension and of laparotomy 16 years before for a prostatic cystoadenoma with diameter of $20 \mathrm{~cm}$ completely excised (Figure 1), who presented to our hospital complaining hypogastric pain since 3 months associated with obstructive voiding symptoms (weak urinary stream with intermittent flow) and constipation. Rectal examination revealed a large elastic mass compressing the right side of the anterior rectal wall. The patient did not report any episode of hematuria, fever or loss of weight. Baseline blood parameters at presentation identified a moderately raised C-reactive protein level with a normal renal biochemistry profile and an elevated serum prostate specific antigen (PSA) of $20.5 \mathrm{ng} / \mathrm{ml}$. A needle core biopsy showed benign prostatic tissue with no evidence of malignancy. Computed tomography (TC) scan showed a $11.6 \times 9 \times 8 \mathrm{~cm}$ multiloculated cystic pelvic mass with a well-defined wall compressing the right side of rectum and with heterogeneous and predominantly peripheral contrast enhancement (Figures 2-5).

An exploratory laparoscopy performed through 3 trocars (12 $\mathrm{mm}$ prerectal right and left and $5 \mathrm{~mm}$ in left iliac fossa) and periumbilical camera, revealed, by using a laparoscopic ultrasound probe, a large multiloculated cystic mass arising from pelvic floor until mesosigma origin; the bladder was displaced anteriorly. 


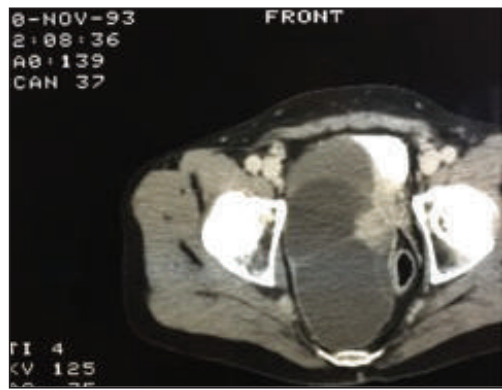

Figure 1.

Previous prostatic

cystadenoma

(16 years before).

\section{Figures 2-5.}

Recurrence of prostatic cystadenoma.

2.

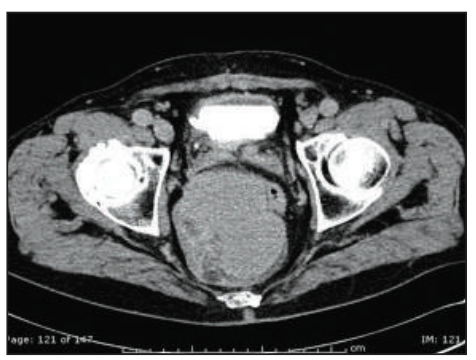

4.

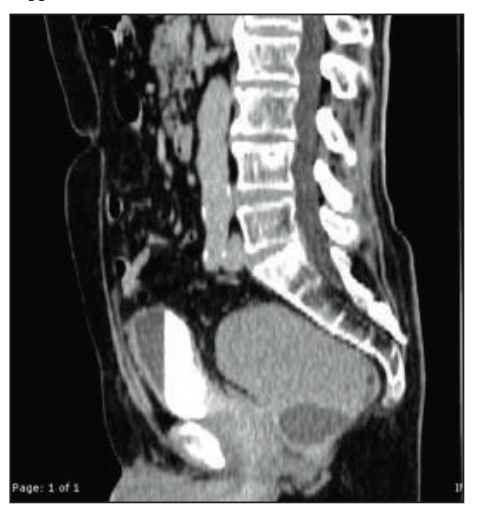

3.

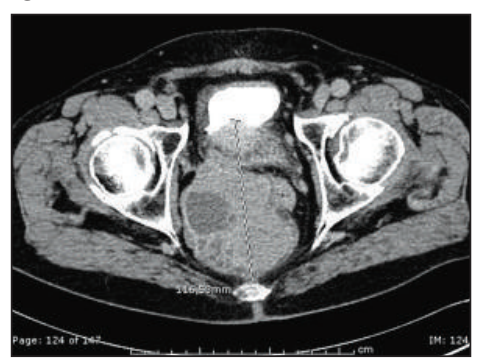

5.

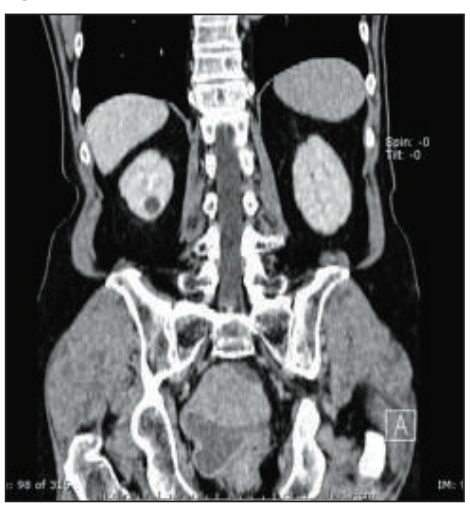

After blunt dissection of the superior part of the cystic mass, we opened it draining more than $150 \mathrm{cc}$ of dense brown liquid, we removed a large dome of the cyst (negative for malignancy at histological examination) and we proceeded with its marsupialization; similar treatment was performed for smaller neighboring cysts. The pathologic findings were consistent with giant multilocular prostatic cystadenoma. At follow up 48 months later, the patient remained free of lower urinary tract symptoms and there were no signs of recurrence.

\section{REFERENCES}

1. Watanabe J, Konishi T, et al. A case of giant prostatic cystadenoma. Hinyokika kiyo. 1990; 36:1077-9.

2. Maluf HM, King ME, et al. Giant multilocular prostatic cystadenoma: a distinctive lesion of the retroperitoneum in men. A report of two cases. Am J Surg Pathol. 1991; 15:131-5.

3. Datta MW, Hosenpud J, et al. Giant multilocular cystadenomaof the prostate responsive to GnRH antagonists. Urology. 2003; 61:225.

Discussion, Tables and Supplementary References are posted on www.aiua.it

\section{Correspondence}

Tiziano Zago, MD (Corresponding Author)

tzago@aogarbagnate.lombardia.it

Davide Abed El Rahman, MD

Giuseppe Verduci, MD

Gianpaolo Baroni, MD

Marco Lorenzo Berardinelli, MD

Urology Department, "Guido Salvini” Hospital, ASST Rhodense,

Corso Europa, 250 - Rho (MI), Italy

\section{Umberto Pea, MD}

Urology Department, "Fatebenefratelli e Oftalmico" Hospital,

ASST Fatebenefratelli Sacco

Via G.B. Grassi, 74 - Milano, Italy

Eugenio Morandi, MD

Marco Castoldi, MD

Surgery Department, "Guido Salvini" Hospital, ASST Rhodense Corso Europa, 250 - Rho (MI), Italy 